\title{
CIÊNCIANATURA
}

\section{A variational formulation for relativistic mechanics based on Riemannian geometry and its application to the quantum mechanics context}

\author{
Fabio Silva Botelho
}

Departamento de Matemática, Universidade Federal de Santa Catarina,Florianópolis, SC - Brazil

\begin{abstract}
This article develops a variational formulation of relativistic nature applicable to the quantum mechanics context. The main results are obtained through basic concepts on Riemannian geometry. Standards definitions such as vector fields and connection have a fundamental role in the main action establishment. In the last section, as a result of an approximation for the main formulation, we obtain the relativistic Klein-Gordon equation.
\end{abstract}

Keywords: Quantum mechanics; Riemannian geometry; Klein-Gordon equation 


\section{Introduction}

In this article we develop a variational formulation suitable for the relativistic quantum mechanics approach in a free particle context. The results are based on fundamental concepts of Riemannian geometry and suitable extensions for the relativistic case. Definitions such as vector fields, connection, Lie Bracket and Riemann tensor are addressed in the subsequent sections for the main energy construction.

Indeed, the action developed in this article, in some sense, generalizes and extends the one presented in the Weinberg book [8], in chapter 12 at page 358. In such a book, the concerned action is denoted by $I=I_{M}+I_{G}$, where $I_{M}$, the matter action, for $N$ particles with mass $m_{n}$ and charge $e_{n}, \forall n \in\{1, \ldots, N\}$, is given by

$$
\begin{aligned}
I_{M}= & -\sum_{n=1}^{N} m_{n} \int_{-\infty}^{\infty} \sqrt{-g_{\mu \nu}(x(p)) \frac{d x_{n}^{\mu}(p)}{d p} \frac{d x_{n}^{\nu}(p)}{d p}} d p \\
& -\frac{1}{4} \int_{\Omega} \sqrt{g} F_{\mu \nu} F^{\mu \nu} d^{4} x \\
& +\sum_{n=1}^{N} e_{n} \int_{-\infty}^{\infty} \frac{d x_{n}^{\mu}(p)}{d p} A_{\mu}(x(p)) d p,
\end{aligned}
$$

where $\left\{x_{n}(p)\right\}$ is the position field with concerning metrics $\left\{g_{\mu \nu}(x(p))\right\}$ and

$$
F_{\mu \nu}=\frac{\partial A_{\nu}}{\partial x_{\mu}}-\frac{\partial A_{\mu}}{\partial x_{\nu}}
$$

represents the electromagnetic tensor field through a vectorial potential $\left\{A_{\mu}\right\}$.

Moreover, the gravitational action $I_{G}$ is defined by

$$
I_{G}=-\frac{1}{16 \pi G} \int_{\Omega} R(x) \sqrt{g} d^{4} x
$$

where

$$
R=g^{\mu \nu} R_{\mu \nu}
$$

Here

$$
R_{\mu \nu}=R_{\mu \sigma \nu}^{\sigma}
$$

where

$$
R_{\mu \sigma \nu}^{\eta}
$$

are the components of the well known Riemann curvature tensor.

According to [8], the Euler-Lagrange equations for $I$ correspond to the Einstein field equations,

$$
R^{\mu \nu}-\frac{1}{2} g^{\mu \nu} R+8 \pi G T^{\mu \nu}=0,
$$

where the energy-momentum tensor $T^{\mu \nu}$ is expressed by

$$
\begin{aligned}
T^{\lambda \kappa}= & \sqrt{g} \sum_{n=1}^{N} \int_{-\infty}^{\infty} \frac{d x_{n}^{\lambda}(p)}{d \tau_{n}} \frac{d x_{n}^{\kappa}(p)}{d \tau_{n}} \delta^{4}\left(x-x_{n}\right) d \tau_{n} \\
& +F_{\mu}^{\lambda}(x) F^{\mu \kappa}(x)-\frac{1}{4} g^{\lambda \kappa} F_{\mu \nu} F^{\mu \nu} .
\end{aligned}
$$


One of the main differences of our model from this previous one, is that we consider a possible variation in the density along the mechanical system.

Also, in our model, the motion of the system in question is specified by a four-dimensional manifold given by the function

$$
\mathbf{r}(\hat{\mathbf{u}}(\mathbf{x}, t))=\left(c t, X_{1}(\mathbf{u}(\mathbf{x}, t)), X_{2}(\mathbf{u}(\mathbf{x}, t)), X_{3}(\mathbf{u}(\mathbf{x}, t))\right),
$$

with corresponding mass density

$$
(\rho \circ \hat{\mathbf{u}}): \Omega \times[0, T] \rightarrow \mathbb{R}^{+},
$$

where $\Omega \subset \mathbb{R}^{3}$ and $[0, T]$ is a time interval. At this point, we define $\phi(\hat{\mathbf{u}}(\mathbf{x}, t))$ as a complex function such that

$$
|\phi(\hat{\mathbf{u}}(\mathbf{x}, t))|^{2}=\frac{\rho(\hat{\mathbf{u}}(\mathbf{x}, t))}{m},
$$

where $m$ denotes the total system mass at rest. We emphasize it seems to be clear that in the previous book the parametrization of the position field, through the parameter $p$, is onedimensional.

In this work we do not consider the presence of electromagnetic fields.

Anyway, the final expression of the related new action here developed is given by

$$
\begin{aligned}
& J(\phi, \mathbf{r}, \hat{\mathbf{u}}, E) \\
= & \int_{0}^{T} \int_{\Omega} m c \sqrt{-g_{i j} \frac{\partial u_{i}}{\partial t} \frac{\partial u_{j}}{\partial t}}|\phi(\hat{\mathbf{u}}(\mathbf{x}, t))|^{2} \sqrt{-g}\left|\operatorname{det}\left(\hat{\mathbf{u}}^{\prime}(\mathbf{x}, t)\right)\right| d \mathbf{x} d t \\
& +\frac{\gamma}{2} \int_{0}^{T} \int_{\Omega} g^{j k} \frac{\partial \phi}{\partial u_{j}} \frac{\partial \phi^{*}}{\partial u_{k}} \sqrt{-g}\left|\operatorname{det}\left(\hat{\mathbf{u}}^{\prime}(\mathbf{x}, t)\right)\right| d \mathbf{x} d t \\
& +\frac{\gamma}{4} \int_{0}^{T} \int_{\Omega} g^{j k}\left(\phi^{*} \frac{\partial \phi}{\partial u_{l}}+\phi \frac{\partial \phi^{*}}{\partial u_{l}}\right) \Gamma_{j k}^{l} \sqrt{-g}\left|\operatorname{det}\left(\hat{\mathbf{u}}^{\prime}(\mathbf{x}, t)\right)\right| d \mathbf{x} d t \\
& +\frac{\gamma}{2} \int_{0}^{T} \int_{\Omega}|\phi|^{2} g^{j k}\left(\frac{\partial \Gamma_{l k}^{l}}{\partial u_{j}}-\frac{\partial \Gamma_{j k}^{l}}{\partial u_{l}}+\Gamma_{l k}^{p} \Gamma_{j p}^{l}-\Gamma_{j k}^{p} \Gamma_{l p}^{l}\right) \sqrt{-g}\left|\operatorname{det}\left(\hat{\mathbf{u}}^{\prime}(\mathbf{x}, t)\right)\right| d \mathbf{x} d t \\
& -\int_{0}^{T} E(t)\left(\int_{\Omega}|\phi(\hat{\mathbf{u}}(\mathbf{x}, t))|^{2} \sqrt{-g}\left|\operatorname{det}\left(\hat{\mathbf{u}}^{\prime}(\mathbf{x}, t)\right)\right| d \mathbf{x} / c-1\right) c d t .
\end{aligned}
$$

Observe that the action part

$$
\begin{aligned}
& \frac{\gamma}{2} \int_{0}^{T} \int_{\Omega}|\phi|^{2} g^{j k}\left(\frac{\partial \Gamma_{l k}^{l}}{\partial u_{j}}-\frac{\partial \Gamma_{j k}^{l}}{\partial u_{l}}+\Gamma_{l k}^{p} \Gamma_{j p}^{l}-\Gamma_{j k}^{p} \Gamma_{l p}^{l}\right) \sqrt{-g}\left|\operatorname{det}\left(\hat{\mathbf{u}}^{\prime}(\mathbf{x}, t)\right)\right| d \mathbf{x} d t \\
= & \frac{\gamma}{2} \int_{0}^{T} \int_{\Omega}|\phi|^{2} \hat{R} \sqrt{-g}\left|\operatorname{det}\left(\hat{\mathbf{u}}^{\prime}(\mathbf{x}, t)\right)\right| d \mathbf{x} d t,
\end{aligned}
$$

where

and

$$
\begin{gathered}
\hat{R}=g^{j k} \hat{R}_{j k}, \\
\hat{R}_{j k}=\hat{R}_{j l k}^{l}
\end{gathered}
$$

$$
\hat{R}_{i j k}^{l}=\frac{\partial \Gamma_{j k}^{l}}{\partial u_{i}}-\frac{\partial \Gamma_{i k}^{l}}{\partial u_{j}}+\Gamma_{j k}^{p} \Gamma_{i p}^{l}-\Gamma_{i k}^{p} \Gamma_{j p}^{l}
$$

represents the Riemann curvature tensor, corresponds tho the Hilbert-Einstein one, as specified in the subsequent sections. 
In the last section, we show how such a formulation may result, as an approximation, the well known relativistic Klein-Gordon one and the respective Euler-Lagrange equations. We believe the main results obtained may be extended to more complex mechanical systems, including in some extent, the quantum mechanics approach.

Finally, about the references, details on the Sobolev Spaces involved may be found in $[1,4]$. For standard references in quantum mechanics, we refer to $[3,6,7]$ and the non-standard [2].

\section{Some Inrodictory topics on vector analysis and Riemannian gemetry}

In this section we present some introductory remarks on Riemannian geometry. The results here developed have been presented in details in [5]. For the sake of completeness, we repeat some of the proofs.

We start with the definition of surface in $\mathbb{R}^{n}$.

Definition 2.1 (Surface in $\mathbb{R}^{n}$, the respective tangent space and the dual one). Let $D \subset \mathbb{R}^{m}$ be an open, bounded, connected set with a regular (Lipschitzian) boundary denoted by $\partial D$. We define a m-dimensional $C^{1}$ class surface $M \subset \mathbb{R}^{n}$, where $1 \leq m<n$, as the range of a function $\mathbf{r}: D \subset \mathbb{R}^{m} \rightarrow \mathbb{R}^{n}$, where

$$
M=\left\{\mathbf{r}(\mathbf{u}): \mathbf{u}=\left(u_{1}, \ldots, u_{m}\right) \in D\right\}
$$

and

$$
\mathbf{r}(\mathbf{u})=\hat{X}_{1}(\mathbf{u}) \mathbf{e}_{1}+\hat{X}_{2}(\mathbf{u}) \mathbf{e}_{2}+\cdots+\hat{X}_{n}(\mathbf{u}) \mathbf{e}_{n},
$$

where $\hat{X}_{k}: D \rightarrow \mathbb{R}$ is a $C^{1}$ class function, $\forall k \in\{1, \ldots, n\}$, and $\left\{\mathbf{e}_{1}, \ldots, \mathbf{e}_{n}\right\}$ is the canonical basis of $\mathbb{R}^{n}$.

Let $\mathbf{u} \in D$ and $p=\mathbf{r}(\mathbf{u}) \in M$. We also define the tangent space of $M$ at $p$, denoted by $T_{p}(M)$, as

$$
T_{p}(M)=\left\{\alpha_{1} \frac{\partial \mathbf{r}(\mathbf{u})}{\partial u_{1}}+\cdots+\alpha_{m} \frac{\partial \mathbf{r}(\mathbf{u})}{\partial u_{m}}: \alpha_{1}, \ldots, \alpha_{m} \in \mathbb{R}\right\}
$$

We assume

$$
\left\{\frac{\partial \mathbf{r}(\mathbf{u})}{\partial u_{1}}, \cdots, \frac{\partial \mathbf{r}(\mathbf{u})}{\partial u_{m}}\right\}
$$

to be a linearly independent set $\forall \mathbf{u} \in D$.

Finally, we define the dual space to $T_{p}(M)$, denoted by $T_{p}(M)^{*}$, as the set of all continuous and linear functionals (in fact real functions) defined on $T_{p}(M)$, that is,

$$
T_{p}(M)^{*}=\left\{f: T_{p}(M) \rightarrow \mathbb{R}: f(\mathbf{v})=\alpha \cdot \mathbf{v}, \text { for some } \alpha \in \mathbb{R}^{n}, \forall \mathbf{v}=v_{i} \frac{\partial \mathbf{r}(\mathbf{u})}{\partial u_{i}} \in T_{p}(M)\right\} .
$$

Theorem 2.2. Let $M \subset \mathbb{R}^{n}$ be a m-dimensional $C^{1}$ class surface, where

$$
M=\left\{\mathbf{r}(\mathbf{u}) \in \mathbb{R}^{n}: \mathbf{u} \in D \subset \mathbb{R}^{m}\right\} .
$$

Let $\mathbf{u} \in D, p=\mathbf{r}(\mathbf{u}) \in D$ and $f \in C^{1}(M)$.

Define $d f: T_{p}(M) \rightarrow \mathbb{R}$ by

$$
d f(\mathbf{v})=\lim _{\varepsilon \rightarrow 0} \frac{(f \circ \mathbf{r})\left(\left\{u_{i}\right\}+\varepsilon\left\{v_{i}\right\}\right)-(f \circ \mathbf{r})\left(\left\{u_{i}\right\}\right)}{\varepsilon},
$$


$\forall \mathbf{v}=v_{i} \frac{\partial \mathbf{r}(\mathbf{u})}{\partial u_{i}} \in T_{p}(M)$

Under such hypotheses,

$$
d f \in T_{p}(M)^{*}
$$

Reciprocally, let $F \in T_{p}(M)^{*}$.

Under such assumption, there exists $f \in C^{1}(M)$ such that

$$
F(\mathbf{v})=d f(\mathbf{v}), \forall \mathbf{v} \in T_{p}(M) .
$$

Proof. Let $\mathbf{v}=v_{i} \frac{\partial \mathbf{r}(\mathbf{u})}{\partial u_{i}} \in T_{p}(M)$.

Thus,

$$
\begin{aligned}
d f(\mathbf{v}) & =\lim _{\varepsilon \rightarrow 0} \frac{(f \circ \mathbf{r})\left(\left\{u_{i}\right\}+\varepsilon\left\{v_{i}\right\}\right)-(f \circ \mathbf{r})\left(\left\{u_{i}\right\}\right)}{\varepsilon} \\
& =\frac{\partial(f \circ \mathbf{r})(\mathbf{u})}{\partial \hat{X}_{j}} \frac{\partial \hat{X}_{j}(\mathbf{u})}{\partial u_{i}} v_{i} \\
& =\nabla f(\mathbf{r}(\mathbf{u})) \cdot \mathbf{v} \\
& =\alpha \cdot \mathbf{v},
\end{aligned}
$$

where

$$
\alpha=\nabla f(\mathbf{r}(\mathbf{u}))
$$

so that $d f \in T_{p}(M)^{*}$.

Reciprocally, assume $F \in T_{p}(M)^{*}$, that is, suppose there exists $\alpha \in \mathbb{R}^{n}$ such that

$$
F(\mathbf{v})=\alpha \cdot \mathbf{v}
$$

$\forall \mathbf{v}=v_{i} \frac{\partial \mathbf{r}(\mathbf{u})}{\partial u_{i}} \in T_{p}(M)$

Define $f: M \rightarrow \mathbb{R}$ by

$$
f(\mathbf{w})=\alpha \cdot \mathbf{w}, \forall \mathbf{w} \in M .
$$

In particular,

$$
f(\mathbf{r}(\mathbf{u}))=\alpha \cdot \mathbf{r}(\mathbf{u})=\alpha_{j} \hat{X}_{j}(\mathbf{u}), \forall \mathbf{u} \in D .
$$

For $p=\mathbf{r}(\mathbf{u}) \in M$ and $\mathbf{v}=v_{i} \frac{\partial \mathbf{r}(\mathbf{u})}{\partial u_{i}} \in T_{p}(M)$, we have

$$
\begin{aligned}
d f(\mathbf{v}) & =\lim _{\varepsilon \rightarrow 0} \frac{(f \circ \mathbf{r})\left(\left\{u_{i}\right\}+\varepsilon\left\{v_{i}\right\}\right)-(f \circ \mathbf{r})\left(\left\{u_{i}\right\}\right)}{\varepsilon} \\
& =\frac{\partial(f \circ \mathbf{r})(\mathbf{u})}{\partial \hat{X}_{j}} \frac{\partial \hat{X}_{j}(\mathbf{u})}{\partial u_{i}} v_{i} \\
& =\alpha_{j} \frac{\partial \hat{X}_{j}(\mathbf{u})}{\partial u_{i}} v_{i} \\
& =\alpha \cdot \mathbf{v}
\end{aligned}
$$

Therefore,

$$
F(\mathbf{v})=d f(\mathbf{v}), \forall \mathbf{v} \in T_{p}(M)
$$

The proof is complete.

At this point, we present the tangential vector field definition, to be addressed in the subsequent results and sections. 
Definition 2.3 (Vector field). Let $M \subset \mathbb{R}^{n}$ be a m-dimensional $C^{1}$ class surface, where $1 \leq$ $m<n$. We define the set of $C^{1}$ class tangential vector fields in $M$, denoted by $\mathcal{X}(M)$, as

$$
\mathcal{X}(M)=\left\{X=X_{i}(\mathbf{u}) \frac{\partial \mathbf{r}(\mathbf{u})}{\partial u_{i}} \in T(M)=\left\{T_{p}(M): p=\mathbf{r}(\mathbf{u}) \in M\right\}\right\},
$$

where $X_{i}: D \rightarrow \mathbb{R}$ is a $C^{1}$ class function, $\forall i \in\{1, \ldots, m\}$.

Let $f \in C^{1}(M)$ and $X \in \mathcal{X}(M)$. We define the derivative of $f$ on the direction $X$ at $\mathbf{u}$, denoted by $(X \cdot f)(p)$, where $p=\mathbf{r}(\mathbf{u})$, as

$$
\begin{aligned}
(X \cdot f)(p) & =d f(X(\mathbf{u})) \\
& =\lim _{\varepsilon \rightarrow 0} \frac{(f \circ \mathbf{r})\left(\left\{u_{i}\right\}+\varepsilon\left\{X_{i}(\mathbf{u})\right\}\right)-(f \circ \mathbf{r})\left(\left\{u_{i}\right\}\right)}{\varepsilon} \\
& =\frac{\partial(f \circ \mathbf{r})(\mathbf{u})}{\partial u_{i}} X_{i}(\mathbf{u}) .
\end{aligned}
$$

The next definition is also very important for this work, namely, the connection one.

Definition 2.4 (Connection). Let $M \subset \mathbb{R}^{n}$ be a m-dimensional $C^{1}$ class surface, where

$$
M=\left\{\mathbf{r}(\mathbf{u}) \in \mathbb{R}^{n}: \mathbf{u} \in D \subset \mathbb{R}^{m}\right\}
$$

and

$$
\mathbf{r}(\mathbf{u})=\hat{X}_{1}(\mathbf{u}) \mathbf{e}_{1}+\cdots+\hat{X}_{n}(\mathbf{u}) \mathbf{e}_{n} .
$$

We define an affine connection on $M$, as a map $\nabla: \mathcal{X}(M) \times \mathcal{X}(M) \rightarrow \mathcal{X}(M)$ such that 1.

$$
\nabla_{f X+g Y} Z=f \nabla_{X} Z+g \nabla_{Y} Z
$$

2.

$$
\nabla_{X}(Y+Z)=\nabla_{X} Y+\nabla_{X} Z
$$

and

3.

$$
\nabla_{X}(f Y)=(X \cdot f) Y+f \nabla_{X} Y
$$

$\forall X, Y, Z \in \mathcal{X}(M), f, g \in C^{\infty}(M)$.

About the connection representation, we have the following result.

Theorem 2.5. Let $M \subset \mathbb{R}^{n}$ be a m-dimensional $C^{1}$ class surface, where

$$
M=\left\{\mathbf{r}(\mathbf{u}) \in \mathbb{R}^{n}: \mathbf{u} \in D \subset \mathbb{R}^{m}\right\}
$$

and

$$
\mathbf{r}(\mathbf{u})=\hat{X}_{1}(\mathbf{u}) \mathbf{e}_{1}+\cdots+\hat{X}_{n}(\mathbf{u}) \mathbf{e}_{n} .
$$

Let $\nabla: \mathcal{X}(M) \times \mathcal{X}(M) \rightarrow \mathcal{X}(M)$ be an affine connection on $M$. Let $\mathbf{u} \in D, p=\mathbf{r}(\mathbf{u}) \in M$ and $X, Y \in \mathcal{X}(M)$ be such that

$$
X=X_{i}(\mathbf{u}) \frac{\partial \mathbf{r}(\mathbf{u})}{\partial u_{i}}
$$

and

$$
Y=Y_{i}(\mathbf{u}) \frac{\partial \mathbf{r}(\mathbf{u})}{\partial u_{i}} .
$$


Under such hypotheses, we have

$$
\nabla_{X} Y=\sum_{i=1}^{m}\left(X \cdot Y_{i}+\sum_{j, k=1}^{m} \Gamma_{j k}^{i} X_{j} Y_{k}\right) \frac{\partial \mathbf{r}(\mathbf{u})}{\partial u_{i}} \in T_{p}(M)
$$

where $\Gamma_{j k}^{i}$ are defined through the relations,

$$
\nabla_{\frac{\partial \mathbf{r}(\mathbf{u})}{\partial u_{j}}} \frac{\partial \mathbf{r}(\mathbf{u})}{\partial u_{k}}=\Gamma_{j k}^{i}(\mathbf{u}) \frac{\partial \mathbf{r}(\mathbf{u})}{\partial u_{i}} .
$$

Proof. Observe that

$$
\begin{aligned}
\nabla_{X} Y & =\nabla_{X_{i} \frac{\partial \mathbf{r}(\mathbf{u})}{\partial u_{i}}}\left(Y_{j} \frac{\partial \mathbf{r}(\mathbf{u})}{\partial u_{j}}\right) \\
& =X_{i} \nabla_{\frac{\partial \mathbf{r}(\mathbf{u})}{\partial u_{i}}}\left(Y_{j} \frac{\partial \mathbf{r}(\mathbf{u})}{\partial u_{j}}\right) \\
& =X_{i}\left(\frac{\partial \mathbf{r}(\mathbf{u})}{\partial u_{i}} \cdot Y_{j}\right) \frac{\partial \mathbf{r}(\mathbf{u})}{\partial u_{j}}+X_{i} Y_{j} \nabla_{\frac{\partial \mathbf{r}(\mathbf{u})}{\partial u_{i}}} \frac{\partial \mathbf{r}(\mathbf{u})}{\partial u_{j}} \\
& =\left(X_{i} \frac{\partial \mathbf{r}(\mathbf{u})}{\partial u_{i}} \cdot Y_{j}\right) \frac{\partial \mathbf{r}(\mathbf{u})}{\partial u_{j}}+X_{i} Y_{j} \Gamma_{i j}^{k} \frac{\partial \mathbf{r}(\mathbf{u})}{\partial u_{k}} \\
& =\sum_{i=1}^{m}\left(X \cdot Y_{i}+\sum_{j, k=1}^{m} \Gamma_{j k}^{i} X_{j} Y_{k}\right) \frac{\partial \mathbf{r}(\mathbf{u})}{\partial u_{i}}
\end{aligned}
$$

The proof is complete.

Remark 2.6. If the connection in question is such that

$$
\Gamma_{j k}^{i}=\frac{1}{2} g^{i l}\left(\frac{\partial g_{k l}}{\partial u_{j}}+\frac{\partial g_{j l}}{\partial u_{k}}-\frac{\partial g_{j k}}{\partial u_{l}}\right)
$$

such a connection is said to be the Levi-Civita one. In the next lines we assume the concerning connection is indeed the Levi-Civita one.

We finish this section with the Lie Bracket definition.

Definition 2.7 (Lie bracket). Let $M \subset \mathbb{R}^{n}$ be a $C^{1}$ class $m$-dimensional surface where $1 \leq$ $m<n$.

Let $X, Y \in \tilde{\mathcal{X}}(M)$, where $\tilde{\mathcal{X}}(M)$ denotes the set of the $C^{\infty}(M)=\cap_{k \in \mathbb{N}} C^{k}(M)$ class vector fields. We define the Lie bracket of $X$ and $Y$, denoted by $[X, Y] \in \tilde{\mathcal{X}}(M)$, by

$$
[X, Y]=\left(X \cdot Y_{i}-Y \cdot X_{i}\right) \frac{\partial \mathbf{r}(\mathbf{u})}{\partial u_{i}}
$$

where

$$
X=X_{i} \frac{\partial \mathbf{r}(\mathbf{u})}{\partial u_{i}}
$$

and

$$
Y=Y_{i} \frac{\partial \mathbf{r}(\mathbf{u})}{\partial u_{i}} .
$$




\section{A relativistic quantum mechanics action}

In this section we present a proposal for a relativistic quantum mechanics action.

Let $\Omega \subset \mathbb{R}^{3}$ be an open, bounded, connected set with a $C^{1}$ class boundary denoted by $\partial \Omega$. Denoting by $c$ the speed of light, in a free volume context, for a $C^{1}$ class function $\mathbf{r}$ and $\hat{\mathbf{u}} \in W^{1,2}\left(\Omega \times[0, T] ; \mathbb{R}^{4}\right)$, let $(\mathbf{r} \circ \hat{\mathbf{u}}): \Omega \times[0, T] \rightarrow \mathbb{R}^{4}$ be a particle position field where

$$
\mathbf{r}(\hat{\mathbf{u}}(\mathbf{x}, t))=\left(c t, X_{1}(\mathbf{u}(\mathbf{x}, t)), X_{2}(\mathbf{u}(\mathbf{x}, t)), X_{3}(\mathbf{u}(\mathbf{x}, t))\right),
$$

with corresponding mass density

$$
(\rho \circ \hat{\mathbf{u}}): \Omega \times[0, T] \rightarrow \mathbb{R}^{+}
$$

where $[0, T]$ is a time interval.

We denote $\hat{\mathbf{u}}: \Omega \times[0, T] \rightarrow \mathbb{R}^{4}$ point-wise as

$$
\hat{\mathbf{u}}(\mathbf{x}, t)=\left(u_{0}(\mathbf{x}, t), \mathbf{u}(\mathbf{x}, t)\right)
$$

where

$$
u_{0}(\mathbf{x}, t)=c t
$$

and

$$
\mathbf{u}(\mathbf{x}, t)=\left(u_{1}(\mathbf{x}, t), u_{2}(\mathbf{x}, t), u_{3}(\mathbf{x}, t)\right),
$$

$\forall(\mathbf{x}, t)=\left(\left(x_{1}, x_{2}, x_{3}\right), t\right) \in \Omega \times[0, T]$.

At this point, we recall to have defined $\phi(\hat{\mathbf{u}}(\mathbf{x}, t))$ as a complex function such that

$$
|\phi(\hat{\mathbf{u}}(\mathbf{x}, t))|^{2}=\frac{\rho(\hat{\mathbf{u}}(\mathbf{x}, t))}{m},
$$

where $m$ denotes the total system mass at rest. Also, we assume $\phi$ to be a $C^{2}$ class function and define

$$
d \tau^{2}=c^{2} d t^{2}-d X_{1}(\mathbf{u}(\mathbf{x}, t))^{2}-d X_{2}(\mathbf{u}(\mathbf{x}, t))^{2}-d X_{3}(\mathbf{u}(\mathbf{x}, t))^{2},
$$

so that the mass differential will be denoted by

$$
\begin{aligned}
d m & =\frac{\rho(\hat{\mathbf{u}}(\mathbf{x}, t))}{\sqrt{1-\frac{v^{2}}{c^{2}}}} \sqrt{-g}\left|\operatorname{det}\left(\hat{\mathbf{u}}^{\prime}(\mathbf{x}, t)\right)\right| d \mathbf{x} \\
& =\frac{m|\phi(\hat{\mathbf{u}}(\mathbf{x}, t))|^{2}}{\sqrt{1-\frac{v^{2}}{c^{2}}}} \sqrt{-g}\left|\operatorname{det}\left(\hat{\mathbf{u}}^{\prime}(\mathbf{x}, t)\right)\right| d \mathbf{x},
\end{aligned}
$$

where $d \mathbf{x}=d x_{1} d x_{2} d x_{3}$ and $\hat{\mathbf{u}}^{\prime}(\mathbf{x}, t)$ denotes the Jacobian matrix of the vectorial function $\hat{\mathbf{u}}(\mathbf{x}, t)$.

Also,

$$
\begin{gathered}
\mathbf{g}_{i}=\frac{\partial \mathbf{r}(\hat{\mathbf{u}})}{\partial u_{i}}, \forall i \in\{0,1,2,3\}, \\
g_{i j}=\mathbf{g}_{i} \cdot \mathbf{g}_{j}, \forall i, j \in\{0,1,2,3\},
\end{gathered}
$$

and

$$
g=\operatorname{det}\left\{g_{i j}\right\}
$$

Moreover,

$$
\left\{g^{i j}\right\}=\left\{g_{i j}\right\}^{-1}
$$




\subsection{The kinetics energy}

Observe that

$$
\begin{aligned}
c^{2}-v^{2} & =-\frac{d \mathbf{r}(\hat{\mathbf{u}})}{d t} \cdot \frac{d \mathbf{r}(\hat{\mathbf{u}})}{d t} \\
& =-\left(\frac{\partial \mathbf{r}(\hat{\mathbf{u}})}{\partial u_{i}} \frac{\partial u_{i}}{\partial t}\right) \cdot\left(\frac{\partial \mathbf{r}(\hat{\mathbf{u}})}{\partial u_{j}} \frac{\partial u_{j}}{\partial t}\right) \\
& =-\frac{\partial \mathbf{r}(\hat{\mathbf{u}})}{\partial u_{i}} \cdot \frac{\partial \mathbf{r}(\hat{\mathbf{u}})}{\partial u_{j}} \frac{\partial u_{i}}{\partial t} \frac{\partial u_{j}}{\partial t} \\
& =-g_{i j} \frac{\partial u_{i}}{\partial t} \frac{\partial u_{j}}{\partial t},
\end{aligned}
$$

where the product in question is generically given by

$$
\mathbf{y} \cdot \mathbf{z}=-y_{0} z_{0}+\sum_{i=1}^{3} y_{i} z_{i}, \forall \mathbf{y}=\left(y_{0}, y_{1}, y_{2}, y_{3}\right), \mathbf{z}=\left(z_{0}, z_{1}, z_{2}, z_{3}\right) \in \mathbb{R}^{4}
$$

and

$$
v=\sqrt{\left(\frac{d X_{1}(\mathbf{u}(\mathbf{x}, t))}{d t}\right)^{2}+\left(\frac{d X_{2}(\mathbf{u}(\mathbf{x}, t))}{d t}\right)^{2}+\left(\frac{d X_{3}(\mathbf{u}(\mathbf{x}, t))}{d t}\right)^{2}} .
$$

The semi-classical kinetics energy differential is given by

$$
\begin{aligned}
d E_{c} & =\frac{d \mathbf{r}(\hat{\mathbf{u}})}{d t} \cdot \frac{d \mathbf{r}(\hat{\mathbf{u}})}{d t} d m \\
& =-\left(\frac{d \tau}{d t}\right)^{2} d m \\
& =-\left(c^{2}-v^{2}\right) d m,
\end{aligned}
$$

so that

$$
\begin{aligned}
d E_{c} & =-m \frac{\left(c^{2}-v^{2}\right)}{\sqrt{1-\frac{v^{2}}{c^{2}}}}|\phi(\hat{\mathbf{u}})|^{2} \sqrt{-g}\left|\operatorname{det}\left(\hat{\mathbf{u}}^{\prime}(\mathbf{x}, t)\right)\right| d \mathbf{x} \\
& =-m c^{2} \sqrt{1-\frac{v^{2}}{c^{2}}}|\phi(\hat{\mathbf{u}})|^{2} \sqrt{-g}\left|\operatorname{det}\left(\hat{\mathbf{u}}^{\prime}(\mathbf{x}, t)\right)\right| d \mathbf{x} \\
& =-m c \sqrt{c^{2}-v^{2}}|\phi(\hat{\mathbf{u}})|^{2} \sqrt{-g}\left|\operatorname{det}\left(\hat{\mathbf{u}}^{\prime}(\mathbf{x}, t)\right)\right| d \mathbf{x} \\
& =-m c \sqrt{-g_{i j} \frac{\partial u_{i}}{\partial t} \frac{\partial u_{j}}{\partial t}}|\phi(\hat{\mathbf{u}})|^{2} \sqrt{-g}\left|\operatorname{det}\left(\hat{\mathbf{u}}^{\prime}(\mathbf{x}, t)\right)\right| d \mathbf{x},
\end{aligned}
$$

and thus, the semi-classical kinetics energy $E_{c}$ is given by

$$
E_{c}=\int_{0}^{T} \int_{\Omega} d E_{c} d t
$$

that is,

$$
E_{c}=-\int_{0}^{T} \int_{\Omega} m c \sqrt{-g_{i j} \frac{\partial u_{i}}{\partial t} \frac{\partial u_{j}}{\partial t}}|\phi(\hat{\mathbf{u}})|^{2} \sqrt{-g}\left|\operatorname{det}\left(\hat{\mathbf{u}}^{\prime}(\mathbf{x}, t)\right)\right| d \mathbf{x} d t
$$




\subsection{The energy part relating the curvature and wave function}

At this point we define an energy part, related to the Riemann curvature tensor, denoted by $E_{q}$, where

$$
E_{q}=\frac{\gamma}{2} \int_{0}^{T} \int_{\Omega} g^{j k} R_{j k} \sqrt{-g}\left|\operatorname{det}\left(\hat{\mathbf{u}}^{\prime}(\mathbf{x}, t)\right)\right| d \mathbf{x} d t
$$

and

$$
R_{j k}=\operatorname{Re}\left[R_{j i k}^{i}(\phi)\right]
$$

Also, generically $\operatorname{Re}[z]$ denotes the real part of $z \in \mathbb{C}$ and $R_{i j k}^{l}(\phi)$ is such that

$$
\begin{aligned}
& \nabla_{\left(\phi \frac{\partial \mathbf{r}(\hat{\mathbf{u}})}{\partial u_{i}}\right)} \nabla_{\frac{\partial \mathbf{r}(\hat{\mathbf{u}})}{\partial u_{j}}}\left(\phi^{*} \frac{\partial \mathbf{r}(\hat{\mathbf{u}})}{\partial u_{k}}\right)-\nabla_{\left(\phi \frac{\partial \mathbf{r}(\hat{\mathbf{u}})}{\partial u_{j}}\right)} \nabla_{\frac{\partial \mathbf{r}(\hat{\mathbf{u}})}{\partial u_{i}}}\left(\phi^{*} \frac{\partial \mathbf{r}(\hat{\mathbf{u}})}{\partial u_{k}}\right) \\
& -\nabla_{\left[\phi \frac{\partial \mathbf{r}(\hat{\mathbf{u}})}{\partial u_{i}}, \frac{\partial \mathbf{r}(\hat{\mathbf{u}})}{\partial u_{j}}\right]}\left(\phi^{*} \frac{\partial \mathbf{r}(\hat{\mathbf{u}})}{\partial u_{k}}\right)=R_{i j k}^{l}(\phi) \frac{\partial \mathbf{r}(\hat{\mathbf{u}})}{\partial u_{l}} .
\end{aligned}
$$

More specifically, we have

$$
\begin{aligned}
& \nabla_{\left(\phi \frac{\partial \mathbf{r}(\hat{\mathbf{u}})}{\partial u_{i}}\right)} \nabla_{\frac{\partial \mathbf{r}(\hat{\mathbf{u}})}{\partial u_{j}}}\left(\phi^{*} \frac{\partial \mathbf{r}(\hat{\mathbf{u}})}{\partial u_{k}}\right) \\
= & \nabla_{\left(\phi \frac{\partial \mathbf{r}(\hat{\mathbf{u}})}{\partial u_{i}}\right)}\left(\frac{\partial \phi^{*}}{\partial u_{j}} \frac{\partial \mathbf{r}(\hat{\mathbf{u}})}{\partial u_{k}}+\phi^{*} \Gamma_{j k}^{l} \frac{\partial \mathbf{r}(\hat{\mathbf{u}})}{\partial u_{l}}\right) \\
= & \phi \frac{\partial^{2} \phi^{*}}{\partial u_{i} \partial u_{j}} \frac{\partial \mathbf{r}(\hat{\mathbf{u}})}{\partial u_{k}}+\phi \frac{\partial \phi^{*}}{\partial u_{j}} \Gamma_{i k}^{p} \frac{\partial \mathbf{r}(\mathbf{u})}{\partial u_{p}} \\
& +\phi \frac{\partial\left(\phi^{*} \Gamma_{j k}^{l}\right)}{\partial u_{i}} \frac{\partial \mathbf{r}(\hat{\mathbf{u}})}{\partial u_{l}} \\
& +|\phi|^{2} \Gamma_{i j}^{l} \Gamma_{i l}^{p} \frac{\partial \mathbf{r}(\hat{\mathbf{u}})}{\partial u_{p}} \\
= & \phi \frac{\partial^{2} \phi^{*}}{\partial u_{i} \partial u_{j}} \delta_{k l} \frac{\partial \mathbf{r}(\hat{\mathbf{u}})}{\partial u_{l}}+\phi \frac{\partial \phi^{*}}{\partial u_{j}} \Gamma_{i k}^{l} \frac{\partial \mathbf{r}(\hat{\mathbf{u}})}{\partial u_{l}} \\
& +\phi \frac{\partial\left(\phi^{*} \Gamma_{j k}^{l}\right)}{\partial u_{i}} \frac{\partial \mathbf{r}(\hat{\mathbf{u}})}{\partial u_{l}} \\
& +|\phi|^{2} \Gamma_{j k}^{p} \Gamma_{i p}^{l} \frac{\partial \mathbf{r}(\hat{\mathbf{u}})}{\partial u_{l}}
\end{aligned}
$$

and similarly

$$
\begin{aligned}
& \nabla_{\left(\phi \frac{\partial \mathbf{r}(\hat{\mathbf{u}})}{\partial u_{j}}\right)} \nabla_{\frac{\partial \mathbf{r}(\hat{\mathbf{u}})}{\partial u_{i}}}\left(\phi^{*} \frac{\partial \mathbf{r}(\hat{\mathbf{u}})}{\partial u_{k}}\right) \\
= & \phi \frac{\partial^{2} \phi^{*}}{\partial u_{i} \partial u_{j}} \delta_{k l} \frac{\partial \mathbf{r}(\hat{\mathbf{u}})}{\partial u_{l}}+\phi \frac{\partial \phi^{*}}{\partial u_{i}} \Gamma_{j k}^{l} \frac{\partial \mathbf{r}(\hat{\mathbf{u}})}{\partial u_{l}} \\
+ & \phi \frac{\partial\left(\phi^{*} \Gamma_{i k}^{l}\right)}{\partial u_{j}} \frac{\partial \mathbf{r}(\hat{\mathbf{u}})}{\partial u_{l}} \\
+ & |\phi|^{2} \Gamma_{i k}^{p} \Gamma_{j p}^{l} \frac{\partial \mathbf{r}(\hat{\mathbf{u}})}{\partial u_{l}}
\end{aligned}
$$


Moreover,

$$
\begin{aligned}
& \nabla_{\left[\phi \frac{\partial \mathbf{r}(\hat{\mathbf{u}})}{\partial u_{i}}, \frac{\partial \mathbf{r}(\hat{\mathbf{u}})}{\partial u_{j}}\right]}\left(\phi^{*} \frac{\partial \mathbf{r}(\hat{\mathbf{u}})}{\partial u_{k}}\right) \\
= & \left.\nabla_{\left(-\frac{\partial \mathbf{r}(\hat{\mathbf{u}})}{\partial u_{j}} \cdot \phi\right)}\right) \frac{\partial \mathbf{r}(\hat{\mathbf{u}})}{\partial u_{i}}\left(\phi^{*} \frac{\partial \mathbf{r}(\hat{\mathbf{u}})}{\partial u_{k}}\right) \\
= & -\nabla_{\left(\frac{\partial \phi}{\partial u_{j}} \frac{\partial \mathbf{r}(\hat{\mathbf{u}})}{\partial u_{i}}\right)}\left(\phi^{*} \frac{\partial \mathbf{r}(\hat{\mathbf{u}})}{\partial u_{k}}\right) \\
= & -\frac{\partial \phi}{\partial u_{j}} \nabla_{\frac{\partial \mathbf{r}(\hat{\mathbf{u}})}{\partial u_{i}}}\left(\phi^{*} \frac{\partial \mathbf{r}(\hat{\mathbf{u}})}{\partial u_{k}}\right) \\
= & -\frac{\partial \phi}{\partial u_{j}} \frac{\partial \phi^{*}}{\partial u_{i}} \frac{\partial \mathbf{r}(\hat{\mathbf{u}})}{\partial u_{k}}-\frac{\partial \phi}{\partial u_{j}} \phi^{*} \nabla_{\frac{\partial \mathbf{r}(\hat{\mathbf{u}})}{\partial u_{i}}} \frac{\partial \mathbf{r}(\hat{\mathbf{u}})}{\partial u_{k}} \\
= & -\frac{\partial \phi}{\partial u_{j}} \frac{\partial \phi^{*}}{\partial u_{i}} \frac{\partial \mathbf{r}(\hat{\mathbf{u}})}{\partial u_{k}}-\frac{\partial \phi}{\partial u_{j}} \phi^{*} \Gamma_{i k}^{l} \frac{\partial \mathbf{r}(\hat{\mathbf{u}})}{\partial u_{l}} \\
= & -\frac{\partial \phi}{\partial u_{j}} \frac{\partial \phi^{*}}{\partial u_{i}} \delta_{k l} \frac{\partial \mathbf{r}(\hat{\mathbf{u}})}{\partial u_{l}}-\frac{\partial \phi}{\partial u_{j}} \phi^{*} \Gamma_{i k}^{l} \frac{\partial \mathbf{r}(\hat{\mathbf{u}})}{\partial u_{l}} .
\end{aligned}
$$

Thus,

$$
\begin{aligned}
R_{i j k}^{l}(\phi)= & \phi \frac{\partial^{2} \phi^{*}}{\partial u_{i} \partial u_{j}} \delta_{k l}+\phi \frac{\partial \phi^{*}}{\partial u_{j}} \Gamma_{i k}^{l}+\phi \frac{\partial\left(\phi^{*} \Gamma_{j k}^{l}\right)}{\partial u_{i}}+|\phi|^{2} \Gamma_{j k}^{p} \Gamma_{i p}^{l} \\
& -\phi \frac{\partial^{2} \phi^{*}}{\partial u_{i} \partial u_{j}} \delta_{k l}-\phi \frac{\partial \phi^{*}}{\partial u_{i}} \Gamma_{j k}^{l}-\phi \frac{\partial\left(\phi^{*} \Gamma_{i k}^{l}\right)}{\partial u_{j}}-|\phi|^{2} \Gamma_{i k}^{p} \Gamma_{j p}^{l} \\
& +\frac{\partial \phi}{\partial u_{j}} \frac{\partial \phi^{*}}{\partial u_{i}} \delta_{k l}+\frac{\partial \phi}{\partial u_{j}} \phi^{*} \Gamma_{i k}^{l} .
\end{aligned}
$$

Simplifying this last result, we obtain

$$
\begin{aligned}
R_{i j k}^{l}(\phi)= & \phi \frac{\partial \phi^{*}}{\partial u_{j}} \Gamma_{i k}^{l}+\phi \frac{\partial\left(\phi^{*} \Gamma_{j k}^{l}\right)}{\partial u_{i}}-\phi \frac{\partial \phi^{*}}{\partial u_{i}} \Gamma_{j k}^{l}-\phi \frac{\partial\left(\phi^{*} \Gamma_{i k}^{l}\right)}{\partial u_{j}} \\
& +|\phi|^{2}\left(\Gamma_{j k}^{p} \Gamma_{i p}^{l}-\Gamma_{i k}^{p} \Gamma_{j p}^{l}\right)+\frac{\partial \phi}{\partial u_{j}} \frac{\partial \phi^{*}}{\partial u_{i}} \delta_{k l}+\frac{\partial \phi}{\partial u_{j}} \phi^{*} \Gamma_{i k}^{l} \\
= & |\phi|^{2}\left(\frac{\partial \Gamma_{j k}^{l}}{\partial u_{i}}-\frac{\partial \Gamma_{i k}^{l}}{\partial u_{j}}+\Gamma_{j k}^{p} \Gamma_{i p}^{l}-\Gamma_{i k}^{p} \Gamma_{j p}^{l}\right) \\
& +\frac{\partial \phi}{\partial u_{j}} \frac{\partial \phi^{*}}{\partial u_{i}} \delta_{k l}+\frac{\partial \phi}{\partial u_{j}} \phi^{*} \Gamma_{i k}^{l} \\
= & |\phi|^{2} \hat{R}_{i j k}^{l} \\
& +\frac{\partial \phi}{\partial u_{j}} \frac{\partial \phi^{*}}{\partial u_{i}} \delta_{k l}+\frac{\partial \phi}{\partial u_{j}} \phi^{*} \Gamma_{i k}^{l},
\end{aligned}
$$

where

$$
\hat{R}_{i j k}^{l}=\frac{\partial \Gamma_{j k}^{l}}{\partial u_{i}}-\frac{\partial \Gamma_{i k}^{l}}{\partial u_{j}}+\Gamma_{j k}^{p} \Gamma_{i p}^{l}-\Gamma_{i k}^{p} \Gamma_{j p}^{l}
$$


represents the Riemann curvature tensor.

At this point, we recall to have defined this energy part by

$$
E_{q}=\frac{\gamma}{2} \int_{0}^{T} \int_{\Omega} R \sqrt{-g}\left|\operatorname{det}\left(\hat{\mathbf{u}}^{\prime}(\mathbf{x}, t)\right)\right| d \mathbf{x} d t
$$

where

$$
R=g^{j k} R_{j k},
$$

and as above indicated, $R_{j k}=\operatorname{Re}\left[R_{j i k}^{i}(\phi)\right]$.

Hence the final expression for the energy (action) is given by

$$
\begin{aligned}
J(\phi, \mathbf{r}, \hat{\mathbf{u}}, E)= & -E_{c}+E_{q} \\
& -\int_{0}^{T} E(t)\left(\int_{\Omega}|\phi(\hat{\mathbf{u}}(\mathbf{x}, t))|^{2} \sqrt{-g}\left|\operatorname{det}\left(\mathbf{u}^{\prime}(\mathbf{x}, t)\right)\right| d \mathbf{x} / c-1\right) c d t
\end{aligned}
$$

where $E(t)$ is a Lagrange multiplier related to the total mass constraint.

More explicitly, the final action (the generalized Einstein-Hilbert one), would be given by

$$
\begin{aligned}
& J(\phi, \mathbf{r}, \hat{\mathbf{u}}, E) \\
= & \int_{0}^{T} \int_{\Omega} m c \sqrt{-g_{i j} \frac{\partial u_{i}}{\partial t} \frac{\partial u_{j}}{\partial t}}|\phi(\hat{\mathbf{u}}(\mathbf{x}, t))|^{2} \sqrt{-g}\left|\operatorname{det}\left(\hat{\mathbf{u}}^{\prime}(\mathbf{x}, t)\right)\right| d \mathbf{x} d t \\
& +\frac{\gamma}{2} \int_{0}^{T} \int_{\Omega} g^{j k} \frac{\partial \phi}{\partial u_{j}} \frac{\partial \phi^{*}}{\partial u_{k}} \sqrt{-g}\left|\operatorname{det}\left(\hat{\mathbf{u}}^{\prime}(\mathbf{x}, t)\right)\right| d \mathbf{x} d t \\
& +\frac{\gamma}{4} \int_{0}^{T} \int_{\Omega} g^{j k}\left(\phi^{*} \frac{\partial \phi}{\partial u_{l}}+\phi \frac{\partial \phi^{*}}{\partial u_{l}}\right) \Gamma_{j k}^{l} \sqrt{-g}\left|\operatorname{det}\left(\hat{\mathbf{u}}^{\prime}(\mathbf{x}, t)\right)\right| d \mathbf{x} d t \\
& +\frac{\gamma}{2} \int_{0}^{T} \int_{\Omega}|\phi|^{2} g^{j k}\left(\frac{\partial \Gamma_{l k}^{l}}{\partial u_{j}}-\frac{\partial \Gamma_{j k}^{l}}{\partial u_{l}}+\Gamma_{l k}^{p} \Gamma_{j p}^{l}-\Gamma_{j k}^{p} \Gamma_{l p}^{l}\right) \sqrt{-g}\left|\operatorname{det}\left(\hat{\mathbf{u}}^{\prime}(\mathbf{x}, t)\right)\right| d \mathbf{x} d t \\
& -\int_{0}^{T} E(t)\left(\int_{\Omega}|\phi(\hat{\mathbf{u}}(\mathbf{x}, t))|^{2} \sqrt{-g}\left|\operatorname{det}\left(\hat{\mathbf{u}}^{\prime}(\mathbf{x}, t)\right)\right| d \mathbf{x} / c-1\right) c d t
\end{aligned}
$$

Where $\gamma$ is an appropriate positive constant to be specified.

\section{Obtaining the relativistic Klein- Gordon equation as an approximation of te previus action}

In particular for the special case in which

$$
\mathbf{r}(\hat{\mathbf{u}}(\mathbf{x}, t))=\hat{\mathbf{u}}(\mathbf{x}, t) \approx(c t, \mathbf{x})
$$

so that

$$
\frac{d \mathbf{r}(\hat{\mathbf{u}}(\mathbf{x}, t))}{d t} \approx(c, 0,0,0)
$$


we would obtain

$$
\mathbf{g}_{0} \approx(1,0,0,0), \mathbf{g}_{1} \approx(0,1,0,0), \mathbf{g}_{2} \approx(0,0,1,0) \text { and } \mathbf{g}_{3} \approx(0,0,0,1) \in \mathbb{R}^{4},
$$

and $\Gamma_{i j}^{k} \approx 0, \forall i, j, k \in\{0,1,2,3\}$.

Therefore, denoting $\phi(\hat{\mathbf{u}}(\mathbf{x}, t)) \approx \phi(c t, \mathbf{x})$ simply by a not relabeled $\phi(\mathbf{x}, t)$, we may obtain

$$
\begin{aligned}
E_{q} / c \approx & \frac{\gamma}{2} \int_{0}^{T} \int_{\Omega}\left(-\frac{1}{c^{2}} \frac{\partial \phi(\mathbf{x}, t)}{\partial t} \frac{\partial \phi^{*}(\mathbf{x}, t)}{\partial t}\right. \\
& \left.+\sum_{k=1}^{3} \frac{\partial \phi(\mathbf{x}, t)}{\partial x_{k}} \frac{\partial \phi^{*}(\mathbf{x}, t)}{\partial x_{k}}\right) d \mathbf{x} d t
\end{aligned}
$$

and

$$
E_{c} / c=m c^{2} \int_{0}^{T} \int_{\Omega}|\phi|^{2} \sqrt{1-v^{2} / c^{2}} \sqrt{-g}\left|\operatorname{det}\left(\hat{\mathbf{u}}^{\prime}(\mathbf{x}, t)\right)\right| d \mathbf{x} d t / c \approx m c^{2} \int_{0}^{T} \int_{\Omega}|\phi(\mathbf{x}, t)|^{2} d \mathbf{x} d t .
$$

Hence, we would also obtain

$$
\begin{aligned}
J(\phi, \mathbf{r}, \hat{\mathbf{u}}, E) / c \approx & \frac{\gamma}{2}\left(\int_{0}^{T} \int_{\Omega}-\frac{1}{c^{2}} \frac{\partial \phi(\mathbf{x}, t)}{\partial t} \frac{\partial \phi^{*}(\mathbf{x}, t)}{\partial t} d \mathbf{x} d t\right. \\
& \left.+\sum_{k=1}^{3} \int_{\Omega} \int_{0}^{T} \frac{\partial \phi(\mathbf{x}, t)}{\partial x_{k}} \frac{\partial \phi^{*}(\mathbf{x}, t)}{\partial x_{k}} d \mathbf{x} d t\right) \\
& +m c^{2} \int_{0}^{T} \int_{\Omega}|\phi(\mathbf{x}, t)|^{2} d \mathbf{x} d t \\
& -\int_{0}^{T} E(t)\left(\int_{\Omega}|\phi(\mathbf{x}, t)|^{2} d \mathbf{x}-1\right) d t .
\end{aligned}
$$

The Euler Lagrange equations for such an energy are given by

$$
\begin{aligned}
& \frac{\gamma}{2}\left(\frac{1}{c^{2}} \frac{\partial^{2} \phi(\mathbf{x}, t)}{\partial t^{2}}-\sum_{k=1}^{3} \frac{\partial^{2} \phi(\mathbf{x}, t)}{\partial x_{k}^{2}}\right) \\
& +m c^{2} \phi(\mathbf{x}, t)-E(t) \phi(\mathbf{x}, t)=0, \text { in } \Omega,
\end{aligned}
$$

where we assume the space of admissible functions is given by $C^{1}(\Omega \times[0, T] ; \mathbb{C})$ with the following time and spatial boundary conditions,

$$
\begin{gathered}
\phi(\mathbf{x}, 0)=\phi_{0}(\mathbf{x}), \text { in } \Omega, \\
\phi(\mathbf{x}, T)=\phi_{1}(\mathbf{x}), \text { in } \Omega, \\
\phi(\mathbf{x}, t)=0, \text { on } \partial \Omega \times[0, T] .
\end{gathered}
$$

Equation (24) is the relativistic Klein-Gordon one.

For $E(t)=E \in \mathbb{R}$ (not time dependent), at this point we suggest a solution (and implicitly related time boundary conditions) $\phi(\mathbf{x}, t)=e^{-\frac{i E t}{\hbar}} \phi_{2}(\mathbf{x})$, where

$$
\phi_{2}(\mathbf{x})=0, \text { on } \partial \Omega \text {. }
$$


Therefore, replacing this solution into equation (24), we would obtain

$$
\left(\frac{\gamma}{2}\left(-\frac{E^{2}}{c^{2} \hbar^{2}} \phi_{2}(\mathbf{x})-\sum_{k=1}^{3} \frac{\partial^{2} \phi_{2}(\mathbf{x})}{\partial x_{k}^{2}}\right)+m c^{2} \phi_{2}(\mathbf{x})-E \phi_{2}(\mathbf{x})\right) e^{-\frac{i E t}{\hbar}}=0,
$$

in $\Omega$.

Denoting

$$
E_{1}=-\frac{\gamma E^{2}}{2 c^{2} \hbar^{2}}+m c^{2}-E,
$$

the final eigenvalue problem would stand for

$$
-\frac{\gamma}{2} \sum_{k=1}^{3} \frac{\partial^{2} \phi_{2}(\mathbf{x})}{\partial x_{k}^{2}}+E_{1} \phi_{2}(\mathbf{x})=0, \text { in } \Omega
$$

where $E_{1}$ is such that

$$
\int_{\Omega}\left|\phi_{2}(\mathbf{x})\right|^{2} d \mathbf{x}=1
$$

Moreover, from (24), such a solution $\phi(\mathbf{x}, t)=e^{-\frac{i E t}{\hbar}} \phi_{2}(\mathbf{x})$ is also such that

$$
\begin{aligned}
& \frac{\gamma}{2}\left(\frac{1}{c^{2}} \frac{\partial^{2} \phi(\mathbf{x}, t)}{\partial t^{2}}-\sum_{k=1}^{3} \frac{\partial^{2} \phi(\mathbf{x}, t)}{\partial x_{k}^{2}}\right) \\
& +m c^{2} \phi(\mathbf{x}, t)=i \hbar \frac{\partial \phi(\mathbf{x}, t)}{\partial t}, \text { in } \Omega .
\end{aligned}
$$

At this point, we recall that in quantum mechanics,

$$
\gamma=\hbar^{2} / m
$$

Finally, we remark this last equation (25) is a kind of relativistic Schrödinger-Klein-Gordon equation.

\section{Conclusion}

This work proposes an action (energy) suitable for the relativistic quantum mechanics context. The Riemann tensor represents an important part of the action in question, but now including the density distribution of mass in its expression. In the last section, we obtain the relativistic Klein-Gordon equation as an aproximation of the main action, under specific properly described conditions.

We believe the results obtained may be applied to more general models, such as those involving atoms and molecules subject to the presence of electromagnetic fields.

Anyway, we postpone the development of such studies for a future research. 


\section{References}

[1] R.A. Adams and J.F. Fournier, Sobolev Spaces, 2nd edn. (Elsevier, New York, 2003).

[2] D. Bohm, A Suggested Interpretation of the Quantum Theory in Terms of Hidden Variables I, Phys.Rev. 85, Iss. 2, (1952).

[3] D. Bohm, Quantum Theory (Dover Publications INC., New York, 1989).

[4] F. Botelho, Functional Analysis and Applied Optimization in Banach Spaces, (Springer Switzerland, 2014).

[5] F. Botelho, Real Analysis and Applications, (Springer Switzerland, 2018).

[6] B. Hall, Quantum Theory for Mathematicians (Springer, New York 2013).

[7] L.D. Landau and E.M. Lifschits, Course of Theoretical Physics, Vol. 5- Statistical Physics, part 1. (Butterworth-Heinemann, Elsevier, reprint 2008).

[8] S. Weinberg, Gravitation and Cosmology, Principles and Applications of the General Theory of Relativity, Wiley and Sons, (Cambridge, Massachusetts, 1972). 